\title{
An Unusual Presentation of Needle Tract Seeding from Colonic Cancer
}

Narendra Pandit, Harjeet Singh", Gautham Krishnamurthy and Verma GR

Department of Surgical Gastroenterology, GGS Medical College and Hospital, Faridkot, Punjab, India

*Corresponding author: Harjeet Singh, Assistant Professor, Department of Surgical Gastroenterology, GGS Medical College and Hospital, Faridkot, Punjab, India, Tel: +919915461323; E-mail: harjeetsingh1982@gmail.com

Received date: Aug 14, 2015, Accepted date: Sep 09, 2015, Publication date: Sep 11, 2015

Copyright: (c) 2015 Pandit N, et al. This is an open-access article distributed under the terms of the Creative Commons Attribution License; which permits unrestricted use; distribution; and reproduction in any medium; provided the original author and source are credited.

\begin{abstract}
Needle tract seeding is a recognized complication of obtaining tissue of intraabdominal malignancy by percutaneous techniques. While the exact mechanism of needle tract seeding is still being hypothesized, the knowledge of this concept has led to guidelines avoiding such techniques in a resectable tumor especially tumors of hepatobiliary origin. Here we present a case of colonic carcinoma with a polypoidal soft tissue mass at the site of needle tract. The needle tract tumor could have probably indicated the poor response and should be considered as a poor prognostic factor. This technique should be avoided in resectable colonic tumors.
\end{abstract}

Keywords: Colon cancer; Needle tract seeding

\section{Introduction}

Intra-abdominal malignancies represent a wide spectrum of lesions with respect to the ease of tissue diagnosis. While the endoscopic guided biopsy of the lesions is possible for the upper and lower gastrointestinal tract tumors, tumors arising from solid organs like pancreas necessitate techniques like endoultrasound guided aspiration cytology. Occasionally tumors might be subjected to percutaneous needle aspiration techniques usually due to difficulty in accessing the tumor by other means. Hepatobiliary malignancies form a prime example of such tumors. The occurrence of needle tract seeding is well recognized complication of percutaneous techniques of obtaining tissues from such lesions [1]. Here we present an interesting and rare case of needle tract seeding of colonic malignancy presenting with polypoidal soft tissue mass.

\section{Case Presentation}

29 years lady presented to the surgical outpatient department with complaints of rapidly increasing lump in upper abdomen for 3 months duration and an exophytic mass over the right iliac fossa for 20 days. The patient gave history of recurrent episodes of colicky abdominal pain followed by abdominal distension. Six months prior to admission patient was evaluated for acute abdominal pain in outside hospital and told to have intra-abdominal lump for which she underwent needle aspiration cytology from right iliac fossa. Details of the procedure were not available. On general examination patient was pale. Abdomen examination revealed large, hard non tender mass of size $15 \times 15 \mathrm{~cm}$ occupying the epigastrium, right hypochondrium, periumbilical and right lumbar region. Another fungating mass of size $4 \times 4 \mathrm{~cm}$ was present in the right iliac fossa confined to the skin (Figure 1). Initial blood investigation was normal except for hemoglobin of $4.8 \mathrm{~g} / \mathrm{dL}$. Contrast enhanced CT Scan (CECT) confirmed the mass to be arising from proximal transverse colon of size $16 \times 14 \mathrm{~cm}$ with an exophytic component having multiple air foci and calcifications. The mass was displacing the distal ileal loops with loss of fat planes and probable infiltration. Note was also made of the lesion having component tracking along the previous needle tract (Figure 2). The clinical stage was T4N1M0 (Stage III).Colonoscopy confirmed mid transverse colon growth beyond which scope was not negotiable. Biopsy of the lesion revealed poorly differentiated adenocarcinoma. After optimization, patient was taken for surgery. Intraoperative, locally advanced tumor of size $18 \times 20 \mathrm{~cm}$ arising from the transverse and ascending colon infiltrating approximately 3 feet of terminal ileum. En-bloc resection of colonic tumor with ileum and rectus muscle involving polypoidal skin tumor and ileocolic anastomosis was done (Figure 3). Postoperatively, patient had wound infection, managed by opening wound, which later improved. Patient was discharged on $14^{\text {th }}$ postoperative period. Histopathological examination revealed adenocarcinoma with neuroendocrine differentiation. Patient received adjuvant chemoradiotherpy. Patient received 6 cycles of FOLFOX regime and concurrent radiation therapy in tumor bed area. At 9 months followup, patient was asymptomatic.

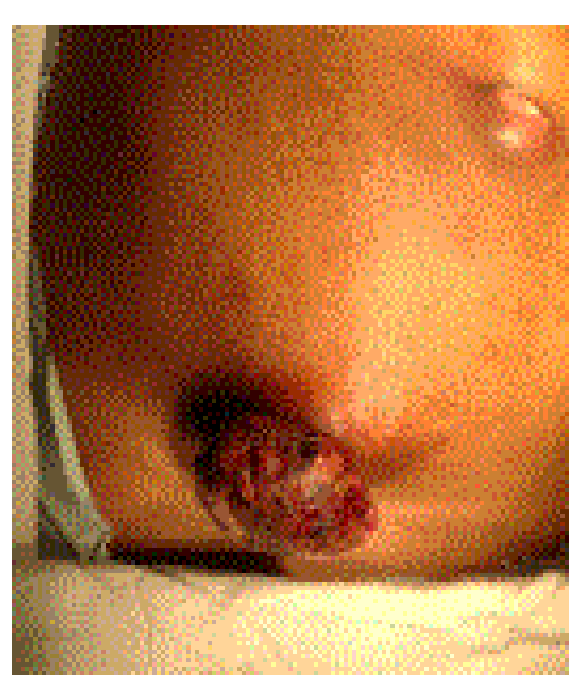

Figure 1: Showing a soft tissue polypoidal tumor at skin surface in right iliac fossa after fine needle aspiration. 


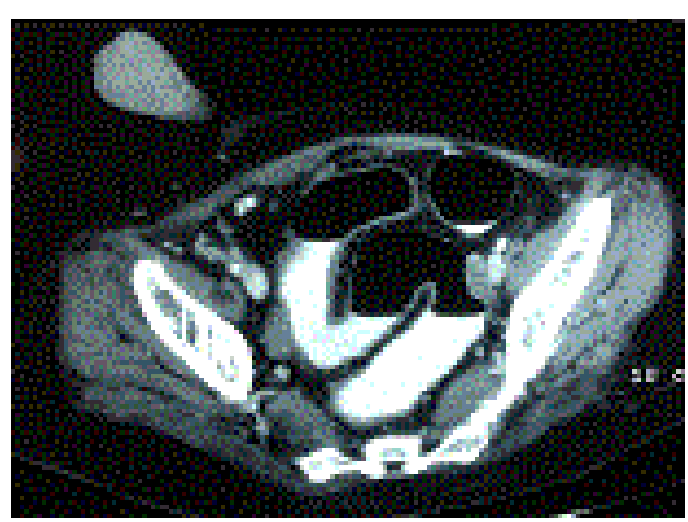

Figure 2: Contrast Enhanced Computed Tomography (CECT) image showing a soft tissue tumor at skin surface corresponding to Figure 1.

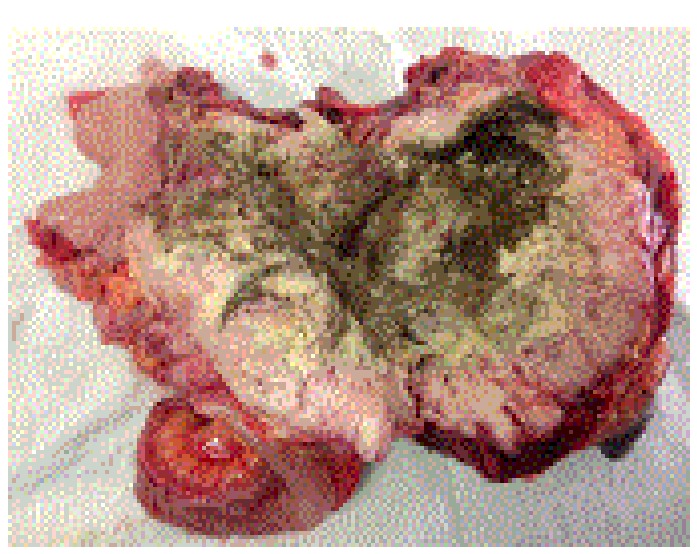

Figure 3: Showing enbloc resected tumor comprising rectus muscle with polypoidal tumor of skin surface and transverse, ascending colon, cecum and ileum. Tumor cut open shows central necrotic tissue with tumor enteric fistula.

\section{Discussion}

The increasing health awareness and improved imaging techniques have led to detection of intraabdominal malignancies at an early stage. Luminal malignancies can be approached through endoscopic means whereas tissue sampling from solid organs remain a challenge. Percutaneous sampling remains a common method to obtain sample where endoluminal ultrasound techniques are not available. Apart from immediate complications related to visceral injury and bleeding, one of the most important late complications is needle tract seeding [1]. Needle tract seeding refers to implantation of tumor cells by contamination when instruments like biopsy needles are used to examine, excise or ablate a tumor [2]. Though it is difficult to accurately estimate the incidence, it has been estimated to be 0 to $4.7 \%$ [3]. These figures are more likely to underestimate the actual incidence since they describe the detection of tumor masses rather than microscopic seeding of the tumor cells.
The mere seeding of the tumor cells is not sufficient to cause recurrence at puncture site. It has to be accompanied by favorable environmental condition for these microscopic cells to proliferate into macroscopic masses. The rate of tumor growth too varies and is also cited as reason for the low incidence for, the patient as to survive for sufficiently long time to manifest the needle site tumor [4].

Such occult seeding could serve as a reservoir of tumor cells contributing to subsequent clinically apparent disease recurrence. This hypothesis is supported by a study that examined the frequency of peritoneal carcinomatosis as a treatment failure pattern in a matched cohort of patients with non-metastatic pancreatic cancer diagnosed by endoscopic ultrasound (EUS) fine needle aspiration (FNA) vs. percutaneous fine needle aspiration [4]. In the EUS FNA group, one patient developed peritoneal carcinomatosis compared with seven in the percutaneous FNA group (2.2 vs. $16.3 \%$; $\mathrm{p}<0.025)$. This study suggests not only a difference in tumor-seeding risk between techniques but also a potentially greater frequency of tumor seeding than previously recognized.

The time period between FNA and detection of needle tract seeding varies from 3 months to 4 years, with a median time being 17 months [3]. Generally, this appears earlier in poorly differentiated tumors and tumors of higher grade. Risk is also increased with use of large diameter needle, multiple passes and the amount of normal parenchyma traversed by the needle [3,5]. In our case, the patient was a young female with aggressive nature of disease, reflected by early needle tract seeding and the neuroendocrine differentiation of adenocarcinoma in histopathological specimen, which itself is a poor prognostic factor [6].

The needle tract seeding in our case presented in the form of soft tissue polypoidal mass from a previous percutaneous aspiration in a case of colonic cancer, which is not reported till date. There is a possibility of the needle tract seeding being actually a form of peritoneal dissemination rather than a mere implantation of tumor cells. This is concordant with the finding that the port site metastasis is commoner in individuals with peritoneal metastasis seeding and the port through which the specimen was extracted. This would imply that both the pathogenesis of peritoneal dissemination as well direct implantation is possible [7]. The present case is the high risk for the local failure. So local radiation therapy was also given to this patient. There is evidence that patient with high risk factor especially fistula and abscess formation had better local control and disease free survival with local radiation therapy [8].

\section{Conclusion}

In the present case, patient underwent needle cytology 6 months before presenting to us. The presentation of needle tract tumor was indicative of the aggressive nature of the tumor. Needle tract seeding although extensively studied in hepatobiliary malignancy, has not been reported with colonic malignancy that also with soft tissue polypoidal type tumor. This is an avoidable complication, as it converts a potentially resectable tumor to metastatic one, because of the tumor spillage and seeding.

\section{References}

1. Tyagi R, Dey P (2014) Needle tract seeding: an avoidable complication. Diagn Cytopathol 42: 636-640. 
Citation: Pandit N, Singh H, Krishnamurthy G, Verma GR (2015) An Unusual Presentation of Needle Tract Seeding from Colonic Cancer. J Nucl Med Radiat Ther 6: 249. doi:10.4172/2155-9619.1000249

Page 3 of 3

2. Ruchika B, Tanya S, Hiremath SS, Prakash K, Nidhi V, et al. (2013) A Study of Fine Needle Aspiration Cytology of Intra-Abdominal Masses In And Around Davangere, Karnataka. JARBS 5: 290-293.

3. Salva MA, Hagab B, Hyde C, Guo B, Buckels JA, et al. (2008) Needle tract seeding following biopsy of liver lesions in the diagnosis of hepatocellular cancer: a systematic review and meta-analysis. Gut 57: 1592-1596.

4. Smith EH (1991) Complications of percutaneous abdominal fine-needle biopsy. Review. Radiology 178: 253-258.

5. Llovet JM, Vilana R, Brú C, Bianchi L, Salmeron JM, et al. (2001) Increased risk of tumor seeding after percutaneous radiofrequency ablation for single hepatocellular carcinoma. Hepatology 33: 1124-129.
6. Sun MH (2004) Neuroendocrine differentiation in sporadic CRC and hereditary nonpolyosis colorectal cancer. Dis Markers 20: 283-288.

7. Ishii H, Okada S, Okusaka T, Yoshimori M, Nakasuka H, et al. (1998) Needle tract implantation of hepatocellular carcinoma after percutaneous ethanol injection. Cancer 82: 1638-1642.

8. Willett CG, Fung CY, Kaufman DS, Efird J, Shellito PC (1993) Postoperative radiation therapy for high-risk colon carcinoma. J Clin Oncol 11: 1112-1117. 